\title{
COMPOSICIÓN, CORRELACIONES DE FUERZA Y ELABORACIÓN DE ESTRATEGIAS EN EL PLENO CONFECH (2011-2015) ${ }^{1}$
}

\author{
Marcelo Mella PolanCO ${ }^{2}$
}

\begin{abstract}
RESUMEN
Este artículo analiza la composición inicial del pleno CONFECH durante el período 2011-2015 y el diseño de estrategias en dicho espacio, considerando que el tipo de alianzas y bloques políticos determina, en buena parte, la gobernanza de la Confederación y sus capacidades para la actuación en la política nacional. Se abordan en este trabajo tres interrogantes principales: ¿Cómo varía la composición anual del Pleno y sus correlaciones iniciales? ¿Qué efectos produce esta composición inicial en la generación de alianzas? ¿Cómo varía la definición de estrategias por parte de las diferentes organizaciones y actores, considerando su posición relativa en este espacio? Para responder estas preguntas se indagará en los efectos mecánicos y político-estratégicos de las composiciones del pleno CONFECH.
\end{abstract}

\section{Palabras Claves: Movimiento Estudiantil, Política Chilena, Conflicto Social, REFORMAS SECTORIALES, DEMOCRATIZACIÓN.}

\section{Composição, Correlações de ForÇa E ElaboraÇão De Estratégias No PLENÁRIO CONFECH (2011-2015)}

\begin{abstract}
RESUMO
Este artigo analisa a composição inicial do plenário CONFECH durante o período 20112015 e o planejamento de estratégias neste espaço, considerando que o tipo de alianças e blocos políticos determina, em grande parte, a governança da Confederação e suas capacidades de ação na política nacional. Neste trabalho são abordadas três questões principais: Como varia a composição anual do Plenário e suas correlações iniciais? Quais são os efeitos que provoca esta composição inicial na geração de alianças? Como varia a definição de estratégias das diferentes organizações e atores, considerando sua posição relativa neste espaço? Para responder estas perguntas indagarei nos efeitos mecânicos e político-estratégicos das composições do plenário CONFECH.

Palavras-Chave: Movimento Estudantil, Política Chilena, Conflito Social, Reformas SETORIAIS, DEMOCRATIZAÇÃO.

\section{COMPOSITION, BALANCE OF POWER AND STRATEGY DEVELOPMENT IN CONFECH PLENARY (2011-2015)}

\footnotetext{
${ }^{1}$ Este artículo ha sido elaborado en el marco de los proyectos FONDECYT Regular, código 1130323 y DICYT Regular, código 031652MP. Como producto, es resultado de una línea de investigación orientada al análisis del movimiento estudiantil universitario chileno durante el período 2010 a 2015. La secuencia de ${ }^{2}$ Académico Departamento de Historia, Universidad de Santiago de Chile; Doctor en Estudios Americanos, Instituto de Estudios Avanzados, Universidad de Santiago de Chile; Magíster en Ciencia Política, Instituto de Asuntos Públicos, Universidad de Chile; Licenciado en Historia, Universidad Católica de Valparaíso. Email: marcelo.mella@usach.cl
} 


\begin{abstract}
This article focuses on the initial composition of CONFECH plenary during the 2011-2015 periods and the development of strategies in such space, taking into account that the governance of the Confederation and its capacity for action in national politics is determined by the kinds of political parties and alliances. Three main questions are addressed in this paper: How does the annual plenary composition, and its initial correlations, vary? What are the effects of this initial composition on building alliances? How does the definition of strategies by different organizations and actors vary, regarding their relative position in this space? This article answers these questions by inquiring into mechanical and political-strategic effects of the compositions of the plenary CONFECH.

Keywords: Student Movement, Chilean Politics, Social Conflict, SeCtoral Reforms, DEMOCRATIZATION.
\end{abstract}

\title{
1. INTRODUCCIÓN
}

La creciente relevancia que ha adquirido el tema educacional en Chile durante los últimos años, convirtió a la Confederación de Estudiantes de Chile (CONFECH) en un actor promotor - frente al Estado - de múltiples demandas referidas al sistema educacional en sus diferentes componentes y dimensiones. Desde los años iniciales del primer gobierno de Bachelet, la reiteración y crecimiento de las movilizaciones de este sector produjo convergencias entre las principales organizaciones que reunieron a los actores de la educación secundaria y universitaria, así como una expansión territorial de nivel nacional de las acciones de protesta.

En una perspectiva orgánica, la CONFECH en su condición de multifederativa, posee como órganos principales la Mesa Ejecutiva y el Pleno. La Mesa Ejecutiva está integrada por los voceros de cada zonal y su tarea principal consiste en comunicar e implementar los acuerdos emanados del Pleno. Por su parte, el Pleno es el espacio de deliberación, acuerdos y toma de decisiones colectiva por parte de las federaciones afiliadas (CONFECH, 2010; 2015).

Este artículo analiza la composición inicial del Pleno y el diseño de estrategias en dicho espacio, considerando que el tipo de alianzas y bloques políticos determina, en buena parte, la gobernanza de la Confederación y sus capacidades para la actuación en la política nacional. Si bien esta perspectiva podría ser calificada de "endógena", porque explica el comportamiento de un actor en base a sus "condiciones internas"; argumentamos en este artículo que este enfoque debe ser considerado "complementariamente" a otras perspectivas inclinadas a explicar el comportamiento de actores por las "ventanas de oportunidad". Se asume, en nuestra visión, que el "peso" o relevancia de cada actor en el Pleno no es un resultado mecánico de la cantidad de federaciones que controla cada fuerza política o bloque, sino de la capacidad (contingente) de las organizaciones, para integrarse en coaliciones ganadoras o, mediante el uso de su capacidad de "chantaje", para direccionar la competencia política (Sartori, 1980: 122-123). Siguiendo a Sartori (1980), utilizamos los conceptos de "capacidad de coalición" (capacidad para constituir o integrarse a una coalición mayoritaria) y "capacidad de chantaje" (capacidad para incidir sobre las tácticas y 
la orientación de la contienda política), como criterios "cualitativos" que permiten determinar la relevancia política de un actor. ${ }^{3}$

Puesto que una primera condición para la gobernanza del espacio es convertir una expresión cuantitativa (número de federaciones) de la fuerza política de cada actor, en una expresión cualitativa (dotada de capacidad de "coalición", "chantaje” y gestión política), se abordan en este trabajo tres interrogantes principales: ¿Cómo varía la composición anual del Pleno y sus correlaciones iniciales? ¿Qué efectos produce esta composición inicial en términos de diseño de estrategias y oportunidades para la generación de alianzas? ¿Cómo varía la definición de estrategias por parte de las diferentes organizaciones y actores considerando su posición relativa en este espacio?

Para responder estas preguntas se indagará en los efectos mecánicos y políticoestratégicos (Tageepera, 2007; Dixit \& Nalebuff, 2010) de las composiciones del pleno CONFECH. Por efectos mecánicos se entienden aquellos que provienen de manera determinista de la composición inicial del Pleno, por ejemplo, la distribución de poder anual y la jerarquía de la triada organizacional que surge de la renovación anual (hasta abril) de las federaciones asociadas. Por efectos político-estratégicos entendemos aquellas creencias básicas que fundamentan las decisiones de los actores y que definen la estimación de costos y beneficios por cada acción política, la estructura de oportunidad y las expectativas de reciprocidad en la interacción en base a la experiencia.

\section{DEFINICIONES DE ENFOQUE Y MÉTODO}

En nuestra perspectiva suponemos que la Multifederativa opera como un particular espacio de construcción sociopolítica desde las organizaciones políticas estudiantiles en interacción. Una de sus principales particularidades consiste en un proceso complejo de institucionalización desde el 2011 en adelante, como respuesta a su crecimiento y mayor centralidad en la política nacional que, sin embargo, no ha logrado revertir la creciente fragmentación y polarización del espacio. Se asume que la Confederación funciona como un "espacio", siguiendo a Pierre Bourdieu (1985; 1999), puesto que constituye un "campo" de lucha y competencia entre actores por los recursos en juego. En este caso, se trata primordialmente de controlar un espacio de producción de narrativas que ha contribuido al desplazamiento del sentido común político en Chile durante los últimos años. Parafraseando a George Simmel (2014), el espacio confederado surge como una construcción social "exclusiva" que posibilita la "coexistencia" (Simmel, 2014: 598), con límites, divisiones internas, desplazamientos y modos de lucha que resultan de la producción de pensamiento racional y abstracto de sus actores (Simmel, 2014: 596-621).

\footnotetext{
${ }^{3}$ En la ciencia política el análisis de la relevancia de los actores políticos ha tenido como precursor a Giovanni Sartori, quien define dos criterios metodológicos destinados a determinar la importancia de los partidos en un sistema, de la siguiente forma:

Regla 1 (Capacidad de coalición). Se puede descontar a un partido menor por su irrelevancia cuando es superfluo en el transcurso del tiempo, en el sentido que nunca se le necesita o se le incluye en alguna coalición mayoritaria viable. Por el contrario, se debe contar a un partido menor, sin importar lo pequeño que sea, si se encuentra en posición de determinar en el transcurso del tiempo, o en algún momento, cuando menos una de las posibles mayorías gobernantes (...).

Regla 2 (Capacidad de chantaje). Un partido es importante siempre que su existencia o creación afecte las tácticas de la contienda partidista, en particular cuando modifica la dirección de la contienda (...), sea hacia la izquierda, la derecha o en ambos sentidos, de los partidos orientados hacia el gobierno (Sartori, 1980: 122123; Mella, 2012: 114-115).
} 
Del mismo modo, nuestro enfoque asume que la construcción de poder desde el espacio de la multifederativa, se caracteriza por tratarse de una tarea altamente contingente y determinada por condiciones políticas generales, como los temas que organizan la contienda y la competencia entre los partidos, los niveles de apoyo y desgaste del gobierno, la capacidad de movilización de la oposición social, su composición y correlaciones de fuerza internas, entre otras. Por tanto, nuestro trabajo explica el comportamiento de los actores en base a su configuración interna y las alternativas de acción que nacen de un escenario político concreto. Se trata de un primer nivel de reconstrucción de los contextos estratégicos que permiten entender las decisiones efectivas de actores situados, evitando los determinismos de algunos análisis históricos o culturales del movimiento estudiantil chileno. Entendemos que este movimiento, en tanto actor político, requiere evitar las inclinaciones esotéricas guiadas por el predominio de creencias emocionales o ideológicas. En este sentido, es deseable que el estudio del movimiento estudiantil no aborde su objeto como algo totalmente único e irrepetible (sin códigos comunes), o como un fenómeno asimilable a los partidos políticos que anula la especificidad de las luchas sociales.

El pleno CONFECH es un espacio originado por la agregación de federaciones afiliadas y que varía anualmente en su composición, donde se construyen las correlaciones políticas del movimiento estudiantil universitario a nivel nacional. Es en esta arena, donde las organizaciones estudiantiles se posicionan y desplazan, establecen interacciones, generan sub-espacios, bloques políticos y definen estrategias que los conectan con el ámbito político institucional o social según sea el caso. La elaboración de estrategias sigue un proceso secuencial; donde el momento cero $\left(\mathrm{M}_{0}\right)$ corresponde al resultado anual de las elecciones federativas afiliadas que determinan una "composición" del espacio; el momento uno $\left(\mathrm{M}_{1}\right)$ corresponde al surgimiento de las correlaciones de fuerza o balances de poder; y el momento dos $\left(\mathrm{M}_{2}\right)$ corresponde a la definición de estrategias por parte de las organizaciones o bloques.

Dentro de este espacio, las organizaciones (p.ej. las Juventudes Comunistas o el Frente de Estudiantes Libertarios) o bloques políticos (p.ej. el "Bloque de Conducción" o el Bloque de la Ultra-Izquierda), se relacionan estratégicamente formando tríadas, vale decir, unidades integradas por tres actores (siendo $\mathrm{A}$ el de mayor poder, $\mathrm{B}$ el intermedio y $\mathrm{C}$ el actor subalterno). Como unidad básica para explicar el comportamiento estratégico, la triada se asocia a una noción del poder como un atributo contingente. El poder no le pertenecería en propiedad a ningún actor en particular, sino que surgiría y evolucionaría por decisiones e interacciones (Caplow, 1974: 11).

Esta perspectiva permite explicar la evolución de espacios con alto nivel de contingencia y variabilidad en las distribuciones de poder. En este marco, una característica principal de las triadas sería que se descomponen o degradan con el paso del tiempo, transformando "la fuerza en debilidad y la debilidad en fuerza" (Caplow, 1974: 15). Además, siguiendo los estudios de Theodore Caplow (1974), las triadas tendrían propensión a transformarse en coaliciones de dos actores contra uno, lo que establece las condiciones mínimas para explicar procesos de cambio dinámico en las correlaciones como es el caso de la multifederativa. Dichas coaliciones pueden ser: conservadoras, cuando mantienen la posición dominante de A y mantienen intacta la jerarquía interna entre los actores; revolucionarias, cuando subvierten la posición dominante de A y trastocan la jerarquía interna entre los actores; e impropias, cuando mantienen posición dominante de A y trastocan la jerarquía interna entre los actores. 
Formalmente, siendo para todos los casos, $\mathrm{A}>\mathrm{B}>\mathrm{C}$ (A mayor que $\mathrm{B}$ y $\mathrm{B}$ mayor que C):

a) Son coaliciones conservadoras aquellas en las que $(A+B)>C$ (coalición $A+B$, mayor que $\mathrm{C}$ );

b) Son coaliciones revolucionarias aquellas en las que $(B+C)>A$ (coalición $B+C$, mayor que A);

c) Son coaliciones impropias aquellas en las que $(\mathrm{A}+\mathrm{C})>\mathrm{B}$ (coalición $\mathrm{A}+\mathrm{C}$, mayor que B).

Para este trabajo utilizamos información cualitativa y cuantitativa con el propósito de reconstruir la dimensión formal e institucional de la Confederación, la evolución de apoyos y representación de las fuerzas políticas, sus modos de interacción y las estrategias para generar coaliciones mayoritarias. Con el propósito de definir y comprender la dimensión institucional hemos consultado el "Reglamento de sala CONFECH", aprobado el 5 de agosto 2010 y la "Propuesta de reglamento de Sala CONFECH", aprobado el 10 de enero del año 2015; instrumentos que definen la orgánica y los procedimientos del Pleno. Con el propósito de describir y analizar la composición y correlaciones del Pleno, hemos utilizado las actas de sesiones CONFECH y se ha elaborado una base de datos para el período 2011 a 2015 que incluye el total anual de federaciones afiliadas, especifica los zonales de pertenencia, fuerza política que lidera la federación respectiva y ubicación ideológica de cada federación (ultraizquierda, izquierda, centro y derecha). Para la identificación y análisis de las estrategias de los actores, hemos consultado los informes anuales y de coyuntura elaborados por diferentes fuerzas políticas y se ha utilizado cuatro entrevistas a informantes claves (ex-presidentes de federaciones y militantes de organizaciones políticas).

\section{TENSIONES CONSTITUTIVAS DEL MOVIMIENTO}

Un punto en el que no existe mayor discrepancia es que el movimiento estudiantil anticipa los cambios e innovaciones del sistema de partidos en Chile. En efecto, durante el siglo veinte, cada clivaje emergente que determinó la competencia y el juego político de los partidos fue impulsado, embrionariamente, desde el campo de la política universitaria (Agacino, 2013; Garretón, 1984; Salazar, 2012). Por ejemplo, la generación de los años 1920, con su idealismo anarquista que reivindicó una posición de autonomía extrema para el movimiento, contribuyó a la superación del sistema oligárquico, a la irrupción de nuevos actores institucionales como "partidos de clase" (PC en 1922 y PS en 1933) y a la reestructuración del régimen político de orientación liberal (Garretón, 1984: 68-76). En la siguiente década (1930), se produjo la pérdida de autonomía y "bolchevización" de las organizaciones estudiantiles, como también, la creciente penetración de los partidos en la arena social, ampliando las bases de apoyo y composición social de los mismos y reestructurando las relaciones entre estado, partidos y organizaciones sociales (Garretón, 1984: 78-85).

Estos momentos iniciales representaron paradigmas históricos competitivos para la acción política del movimiento estudiantil, así como, esquemas contrapuestos de interpretación y análisis de la realidad sociopolítica. De este modo, se distinguen dos concepciones antagónicas respecto de la orientación política del movimiento: por una parte, quienes sostienen que los cambios se generan como resultado de la disputa por el sentido 
común y la hegemonía cultural (partidarios de la autonomía del movimiento) y, por otra, quienes postulan que las transformaciones sólo son posibles ocupando espacios de poder institucional o influyendo directamente sobre ellos (partidarios de la "politización" del movimiento). En esta última perspectiva, el desafío consistió en conjugar la doble necesidad de reivindicar creencias propias destinadas a la transformación de la sociedad y maximizar el impacto efectivo del movimiento frente al gobierno y las políticas.

Desde la década de 1950 hasta el quiebre de la democracia en 1973, las organizaciones estudiantiles universitarias tendieron a reflejar, sino a reproducir las dinámicas del sistema de partidos chileno y las principales directrices de la política regional. Con la organización de la CUT en 1953, se profundiza la articulación obreroestudiantil, adquiriendo esta alianza gran protagonismo en la resistencia a Ibáñez y Alessandri. A partir de 1964, con la llegada del gobierno democristiano de Frei Montalva, la reforma universitaria se convierte en la principal manifestación de la lucha antioligárquica del movimiento. Este proceso, sin embargo, quedará enervado por la implosión del movimiento producto de las diferencias programáticas y estratégicas entre las juventudes políticas de la democracia cristiana, el partido socialista y el partido comunista (Garretón, 1984: 86-95).

Durante la dictadura de Pinochet, el movimiento social y la organización estudiantil, como una parte de él, se reconstituye sin mayor referencia a los partidos y en total oposición a los aparatos de la estatalidad autoritaria. Este hecho, hizo que las organizaciones estudiantiles articularan la resistencia a la dictadura desde el espacio socialpopular y en contra de lo estatal-institucional, que aparece como representación de un régimen político brutalmente opresor e ilegítimo. La resistencia de una generación se transformó en un horizonte de experiencia compartida y un repertorio simbólico que definió identitariamente a los colectivos estudiantiles universitarios. La lucha contra la dictadura posibilitó, inicialmente, definir una posición de contestación cultural y política frente al orden social construido por el régimen de Pinochet para posteriormente, a través de una red de organizaciones de base, conquistar espacios de autonomía en la resistencia a la violencia estatal. Estos espacios aparecieron en un primer momento como colectivos "culturales" que disputaron intensamente, durante la década de 1980, la construcción del orden social (Muñoz, 2009; 2011: 120).

Con el fin de la Dictadura, los partidos de la coalición gobernante expanden su presencia en el espacio universitario, controlando durante los gobiernos de Patricio Aylwin y Eduardo Frei Ruz Tagle, un alto número de federaciones de las instituciones pertenecientes al CRUCH. Esta tendencia se interrumpe y revierte con la coyuntura generativa del movimiento estudiantil a fines de los años 90 (movilización 1997 por Ley Marco y creación de ACES durante el 2000) y la articulación nacional del movimiento a comienzos de los años 2000 ("Mochilazo" durante el 2001 [ACES], "Pingüinazo" durante el 2006 [ACES] y movilización 2008 [ACES+CONFECH]) (Mella, 2016: 132-135).

Bajo las condiciones de los cuatro gobiernos concertacionistas, las organizaciones que dieron vida al movimiento estudiantil definieron, en base a sus experiencias históricas, diferentes alternativas para la acción política, cada año durante el primer semestre. En el ámbito de las organizaciones estudiantiles universitarias, la Confederación de Estudiantes de Chile constituyó el espacio donde las organizaciones se posicionaron, interactuaron con otras equivalentes, maximizando su incidencia sobre las políticas públicas sectoriales. Este estudio se concentra en el período transcurrido entre 2011 a 2015, lapso en el que gobiernan 
los presidentes Sebastián Piñera (2009-2013) y Michelle Bachelet en su segunda administración (2014-2018).

Aunque no es el propósito de este artículo, vale la pena decir provisoriamente que, no obstante la actuación de estos gobiernos y sus respectivos niveles de apoyos (electoral y en la opinión pública), en ningún caso estas dimensiones representan explicaciones suficientes, considerando que el análisis de la documentación arroja como componentes del repertorio estudiantil una compleja mixtura de demandas por políticas públicas, crítica a los sistemas de reproducción social y reivindicaciones culturales.

El espacio CONFECH ha sido una arena de operación de alta complejidad, debido a la rápida renovación de liderazgos en cada federación, a las presiones e intentos de cooptación de actores externos y a la creciente centralidad que ha tenido el debate sobre las políticas educacionales desde las coyunturas de 2006, 2008 y 2011. Como proceso de institucionalización, las organizaciones que integran la $\mathrm{CONFECH}$ desarrollaron diferentes trayectorias que las ubicaron más próximos a la arena social o a la política, en base a aprendizajes en cada subcultura. Esta heterogeneidad estructural definió los contornos de su complejo proceso de institucionalización, con reglas del juego mínimas y, en algunos casos, con la "fuerza normativa de lo fáctico".

Se ha preferido hablar de "espacio" connotando el hecho de que se alternan prácticas formales e informales, considerando las creencias e intereses de las diferentes organizaciones políticas. Como se ha señalado, esta noción de "espacio político" se asemeja a la noción de "campo", como espacio social en el que los actores luchan y compiten por los beneficios y recursos; y en el que la posición de cada uno está determinada por la posesión de capital. Hemos optado por el término "espacio político", debido a que las organizaciones del pleno CONFECH tienen trayectorias diversas respecto a lo político institucional. Respecto de los actores externos, el espacio se comporta como un vórtice donde convergen distintas fuerzas e intereses que, a pesar de tener la capacidad de concentrar el juego de las organizaciones estudiantiles, puede operar bajo ciertas condiciones como gatillador de contestación social frente a las políticas.

En relación a las trayectorias de las organizaciones, sus procesos se encuentran marcados por movimientos imprevisibles, determinados por la contingencia y por una gran variedad de fuerzas formales o informales. Por tal razón, no debe entenderse que la evolución de las organizaciones que participan, se desarrolla de manera lineal. Para entender la trayectoria de una organización debemos suponer que se trata de un proceso errático, aleatorio y estocástico, aunque su desplazamiento sea representado de manera lineal.

Siguiendo el cuadro de doble entrada elaborado por Gerardo Munck (1995) (Cuadro $\mathrm{N}^{\circ}$ 1), las organizaciones estudiantiles pueden evolucionar en tres direcciones: i) movimiento políticamente orientado, ii) fuerza política populista y iii) fuerza social comunal (Munck, 1995: 32-36). El eje vertical distingue las arenas de operación, a saber: la arena política institucional y la sociedad civil. El eje horizontal distingue si existe (o no) consistencia entre identidad y estrategia. Se entiende que esta distinción resulta crucial para explicar la posición de un actor, sus desplazamientos y trayectorias específicas, puesto que, una estrategia ofensiva o el predominio de la estrategia sobre la identidad, posiciona a un actor en la arena política institucional. En sentido opuesto, cuando existe una organización con estrategia defensiva, auto restringida y predomina la identidad sobre la estrategia, el actor se ubica en la arena de la sociedad civil. 


\begin{tabular}{|c|c|c|c|}
\hline \multirow{4}{*}{$\begin{array}{l}\text { Arena de } \\
\text { operación }\end{array}$} & & \multicolumn{2}{|c|}{ Consistencia entre identidad y estrategia } \\
\hline & & Sí & No \\
\hline & $\begin{array}{c}\text { Política } \\
\text { Institucional }\end{array}$ & $\begin{array}{l}\text { Movimiento social } \\
\text { políticamente } \\
\text { orientado (Estrategia } \\
\text { ofensiva) }\end{array}$ & $\begin{array}{lr}\text { Fuerza } & \text { política } \\
\text { populista } & \text { (Estrategia } \\
\text { prevalece } & \text { sobre } \\
\text { identidad: pérdida de } \\
\text { autonomía) }\end{array}$ \\
\hline & $\begin{array}{l}\text { Sociedad } \\
\text { Civil }\end{array}$ & $\begin{array}{l}\text { Movimiento social } \\
\text { autor restringido } \\
\text { (Estrategia defensiva) }\end{array}$ & $\begin{array}{l}\text { Fuerza social comunal } \\
/ \text { fundamentalista } \\
\text { (Identidad prevalece } \\
\text { sobre estrategia: } \\
\text { tendencia } \\
\text { ensimismamiento) }\end{array}$ \\
\hline
\end{tabular}

Fuente: Extraído de Munck, 1995.

De esta manera, los actores sociales enfrentan una contradicción constitutiva: el dilema entre identidad y estrategia. Un movimiento social, por definición, debe constituirse en un actor que reivindica, con creencias propias, un proceso de cambio o ruptura en las condiciones de dominación, esto constituye su marco identitario básico. No es posible que un actor sea un movimiento social si no impulsa o promueve, de forma autónoma, un proyecto propio de transformación social. Sin embargo, para cumplir este propósito el actor debe resolver cómo generar impactos concretos en el estado y las políticas. Esto obliga a los actores sociales, cualquiera sea el caso, a plantearse el problema estratégico como un desafío relevante. Considerando estas tensiones, los actores deben administrar un dilema doble: i) En que arena de operación ubicarse y ii) Como construir consistencia entre identidad y estrategia (Munck, 1995).

\section{RECONSTRUYENDO LAS FUERZAS POLÍTICAS DEL ESPACIO}

Con el propósito de ubicar a las orgánicas políticas CONFECH, se ha dividido este espacio político en cuatro posiciones o sectores: derecha, centro, izquierda y ultraizquierda. Cada una de estas ubicaciones se define mediante la posición del actor en una arena de operación y de acuerdo al tipo de equilibrio entre identidad y estrategia.

Como se observa en el cuadro siguiente (Cuadro $\left.\mathrm{N}^{\circ} 2\right)$, el sector de la derecha reúne a aquellos actores partidarios de responder a los problemas de la educación superior mediante un perfeccionamiento de la regulación y mayor financiamiento a las universidades. Los actores de este sector se caracterizan por mantener vínculos con los partidos de la derecha o aquellos pertenecientes al polo conservador de la Nueva Mayoría (Identidad legitimadora + estrategia de integración). Entre sus organizaciones se cuentan: Gremialistas, Acción Liberal (AL) y la Juventud Demócrata Cristiana (JDC).

El sector de centro reúne a organizaciones que apoyan el programa de la Nueva Mayoría en lo concerniente a las reformas sectoriales de educación. Estos actores se caracterizan por una doble estrategia que busca influir en las reformas a través de la disputa interna dentro de la coalición de gobierno y la presión externa del movimiento estudiantil (Identidad de proyecto + estrategia de articulación). Las principales organizaciones de este 
sector son: Juventudes Comunistas (JJCC), Juventudes Socialistas (JS), Juventud Partido por la Democracia (JPPD) y la Nueva Acción Universitaria (NAU).

La izquierda agrupa a organizaciones que buscan avanzar en mayores grados de democratización institucional, a partir de la estrategia de movilización de masas y el diálogo con presión frente a las autoridades de gobierno (Identidad de proyecto + estrategia de ruptura). Los actores que predominan en este sector son: Unión Nacional Estudiantil (UNE), Frente de Estudiantes Libertarios (FEL) e Izquierda Autónoma (IA).

En último lugar, la ultra-izquierda aglutina a organizaciones fundamentalmente auto limitadas, cuyo proyecto básico busca instalar clivajes fuertemente polarizados para levantar un movimiento de carácter insurreccional (Identidad de resistencia + estrategia de insurrección). Los actores más relevantes en este sector durante los últimos cinco años han sido: Movimiento Popular Guachuneit (MPG), Grupos Acción Popular (GAP), Movimiento de Izquierda Revolucionario (MIR), Frente Estudiantil Revolucionario (FER), Juventud Guevarista (JG), Partido de Trabajadores Revolucionarios (PT), Fuerza Universitaria Rebelde (FUR), Juventud Rebelde (JR) y la Corriente Crítica Universitaria (CCU).

Cuadro N²: Posicionamiento de actores CONFECH (2011 - 2015)

\begin{tabular}{|c|c|c|}
\hline POSICION & DESCRIPTORES & ORGANIZACIONES \\
\hline DERECHA & $\begin{array}{l}\text { Identidad Legitimadora }+ \\
\text { Estrategia de Integración: }\end{array}$ & $\begin{array}{l}\text { Acción Liberal (AL) } \\
\text { Juventud Demócrata Cristiana (JDC) } \\
\text { Gremialistas }\end{array}$ \\
\hline CENTRO & $\begin{array}{l}\text { Identidad de Proyecto } \\
\text { (reformas sectoriales) }+ \\
\text { Estrategia de Articulación: }\end{array}$ & $\begin{array}{l}\text { Juventudes Comunistas (JJCC) } \\
\text { Juventudes Socialistas (JS) } \\
\text { Juventud Partido por la Democracia } \\
\text { (JPPD) } \\
\text { Nueva Acción Universitaria (NAU) }\end{array}$ \\
\hline IZQUIERDA & $\begin{array}{l}\text { Identidad de Proyecto } \\
\text { (reformas régimen político) + } \\
\text { Estrategia de Ruptura: }\end{array}$ & $\begin{array}{l}\text { Unión Nacional Estudiantil (UNE) } \\
\text { Frente de Estudiantes Libertarios (FEL) } \\
\text { Izquierda Autónoma (IA) }\end{array}$ \\
\hline $\begin{array}{c}\text { ULTRA- } \\
\text { IZQUIERDA }\end{array}$ & $\begin{array}{l}\text { Identidad de Resistencia + } \\
\text { Estrategia de Insurrección: }\end{array}$ & $\begin{array}{l}\text { Movimiento Popular Guachuneit (MPG) } \\
\text { Grupos Acción Popular (GAP) } \\
\text { Movimiento de Izquierda } \\
\text { Revolucionario (MIR) } \\
\text { Frente Estudiantil Revolucionario (FER) } \\
\text { Juventud Guevarista (JG) } \\
\text { Partido de Trabajadores Revolucionarios } \\
\text { (PT) } \\
\text { Fuerza Universitaria Rebelde (FUR) } \\
\text { Juventud Rebelde (JR) } \\
\text { Corriente Crítica Universitaria (CCU) }\end{array}$ \\
\hline
\end{tabular}

Fuente: Elaboración en base a informes de organizaciones políticas y actas CONFECH (2011-2015)

Considerando el esquema elaborado por Munck (1995) y los criterios para posicionar a los actores del espacio $\mathrm{CONFECH}$, se puede sostener que la tríada básica que permite desde 2011 la administración y gobernanza de la Confederación, está constituida 
por el bloque Centro, Izquierda y Ultraizquierda. Cada uno de estos bloques, a su vez, puede ser analizado internamente como un espacio (sub-espacio), con recursos, lógicas y actores propios, que compiten por la mejor distribución del poder. Sin embargo, para este trabajo solo analizaremos la relación entre correlaciones iniciales del espacio y coaliciones probables considerando la fuerza de cada bloque (estimada por el porcentaje de federaciones asociadas) y su posicionamiento estratégico.

Cuadro $\mathrm{N}^{\circ}$ 3: Posicionamiento estratégico bloques

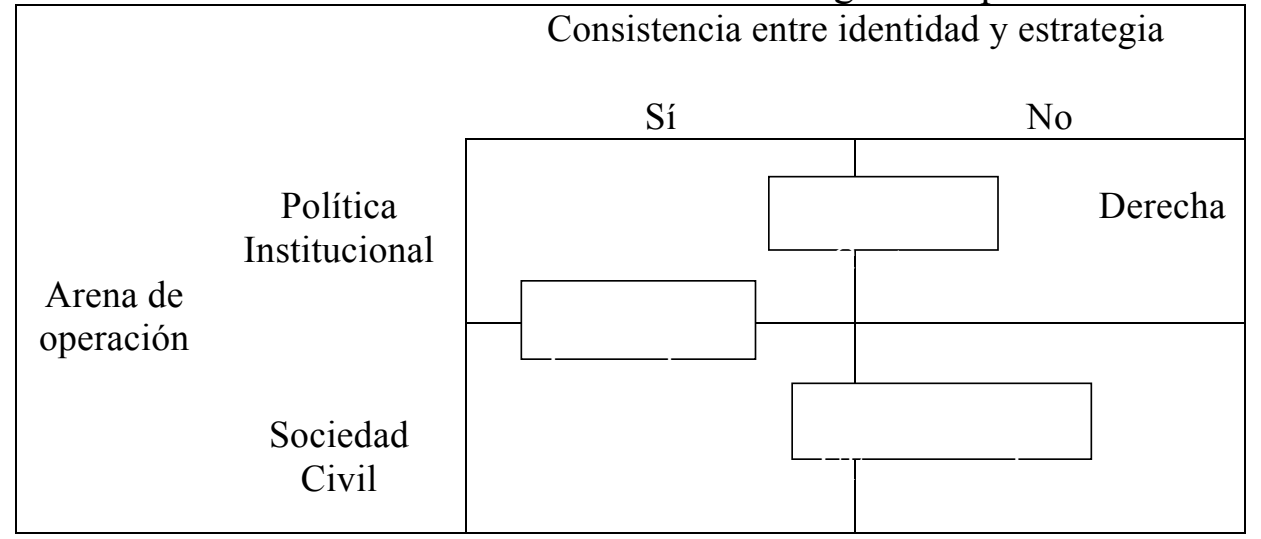

Fuente: Elaboración propia en base a Munck, 1995; Informes de organizaciones políticas (2011 a 2015).

Estratégicamente, el bloque de centro se ubica en la arena político institucional y posee una consistencia relativa entre identidad y estrategia. Esta posición representa una fórmula de equilibrio precario que expone a los actores que pertenecen a esta posición a la pérdida de autonomía y a la cooptación por partidos con representación parlamentaria y otros actores externos. El bloque de izquierda, a su vez, se localiza en una zona de transición entre la sociedad civil y la política institucional; caracterizándose además por un equilibrio relativamente estable entre identidad y estrategia. Esta posición se encuentra restringida por la proyección limitada de los actores hacia la política institucional, situación que aunque no afecta a todas las organizaciones por igual, produce divisiones al interior del sector. Por su parte, el bloque de ultraizquierda se localiza en su conjunto en la arena de la sociedad civil, con un desequilibrio en sus actores caracterizado por el predominio de la identidad por sobre la capacidad estratégica. En esta perspectiva se señala que la ultraizquierda es un bloque integrado por organizaciones auto limitadas en lo político, aunque en este caso, son nítidas las diferencias estratégicas entre actores, situación que tensiona la interna de este conjunto. Respecto de este último bloque, el estudio detallado de su composición interna releva dos situaciones a considerar en el análisis: i) no se puede analizar a priori a la "ultra" en base a la sumatoria de las fuerzas de cada organización, por cuanto existen diferencias tácticas y estratégicas relevantes entre las organizaciones, ii) se observa como dato crucial, la organización política que asume la conducción de la radicalidad sectorial y la intermediación con otros sectores políticos o sociales.

\section{CORRELACIONES DE FUERZA Y TENDENCIAS EN EL ESPACIO}

Las variaciones en la composición anual del espacio se producen como resultado de las elecciones en cada una de las federaciones asociadas, dichas coyunturas, tal como se 
observa en el gráfico siguiente, permiten distinguir algunas tendencias para el período 2011 a 2015:

a) Caída de independientes. Durante estos cinco años se produce el surgimiento de un importante número de organizaciones estudiantiles de contestación social sin adscripción ni vínculos formales con partidos políticos. Se trata de actores que reciben esta denominación porque corresponden a organizaciones articuladas localmente o constituyen colectivos no adscritos a organizaciones de carácter "nacional". La caída desde un 31\% (2011) a un 10\% (2015) se explica, ya sea porque estos referentes constituyen orgánicas estables o porque, en cierta forma, empiezan a generar lineamientos estratégicos comunes, que aunque mínimos, permiten convergencias para la movilización anual y la gestión política del pleno CONFECH (Informes de organizaciones políticas, 2011-2015; Actas CONFECH, 20112015) (Mella M. Ríos H. y Rivera R., 2015b).

b) Continuidad de la derecha como actor marginal. El bloque de derecha durante el período, creció desde un 5,1\% (2011) a un 14\% (2015), resultado que aun cuando representa un $175 \%$ de crecimiento, no permite al sector convertirse en un actor con mayor capacidad estratégica en el contexto del espacio (Informes de organizaciones políticas, 2011-2015; Actas CONFECH, 2011-2015). Como ocurre en el caso de la derecha a nivel de sistema de partidos, el sector aparece además fragmentado por el clivaje ideológico entre integristas católicos y liberales, limitando su capacidad de convergencia y articulación interna (Mella M. Ríos H. y Rivera R., 2015c).

c) Caída del centro. Los datos muestran una drástica caída del bloque de centro desde un $46,2 \%$ (2011) a un 19,6\% (2015), situación que genera un conjunto de consecuencias en el funcionamiento y gestión del Pleno, tales como, desplazamientos en la conducción del movimiento, polarización del espacio y retracción de las orgánicas de las juventudes de partidos de la Concertación y posteriormente de la Nueva Mayoría, en especial, la pérdida creciente de federaciones por parte de la JS y luego de la JJCC (Informes de organizaciones políticas, 2011-2015; Actas CONFECH, 2011-2015) (Mella M. Ríos H. y Rivera R., 2015c).

d) Aumento de la izquierda. Se observa un crecimiento importante de las organizaciones pertenecientes a este sector desde un 10,3\% (2011) a un 45,1\% (2015), lo que revela el recambio en la conducción desde el año 2012 y la perdida de anclaje social de los partidos de clase tradicionales (Informes de organizaciones políticas, 2011-2015; Actas CONFECH, 2011-2015). Si bien, una mirada fina sobre la evolución de este sector, denominado "bloque de conducción", requeriría profundizar en las interacciones y disputas internas entre los tres actores que integran este bloque (IA, FEL y UNE), la constatación de que el bloque se organiza como una tríada, permite concluir preliminarmente que, internamente, las correlaciones de fuerza se constituyen de manera contingente y dinámica. Esta condición confiere flexibilidad y adaptabilidad al bloque para generar expectativas de cambio bajo cierta continuidad de determinadas orgánicas, así como capacidad de intermediación con las fuerzas políticas más radicalizadas (Mella M. Ríos H. y Rivera R., 2015c).

e) Crecimiento ultraizquierda. En el período en estudio se produce un crecimiento de las orgánicas pertenecientes a la ultraizquierda desde un 7,7\% en 2011 a 13,7\% en 2015 
(Informes de organizaciones políticas, 2011-2015; Actas CONFECH, 2011-2015). Este bloque se encuentra integrado por una gran diversidad de actores con orientaciones leninistas, anarquistas, trotskistas y, en algunos casos, nacidos bajo impulsos formativos iniciales de antiguos militantes del MIR o el FPMR, entre otros. Por esta razón aparece como un sector estratégicamente fragmentado y sus fuerzas difícilmente logran un adecuado nivel de articulación y cooperación; motivo por el que, sólo en ciertos contextos, estas fuerzas pueden ser analizadas en forma aditiva. A pesar de lo anterior, estos actores constituyen frecuentemente un "capital de reserva" para las organizaciones situadas en la izquierda, incidiendo de manera relevante en la composición y actuación del Pleno (Mella M. Ríos H. y Rivera R., 2015a, 2015b).

\section{Grafico $\mathrm{N}^{\circ} 1$ : Variaciones composición anual del espacio CONFECH}

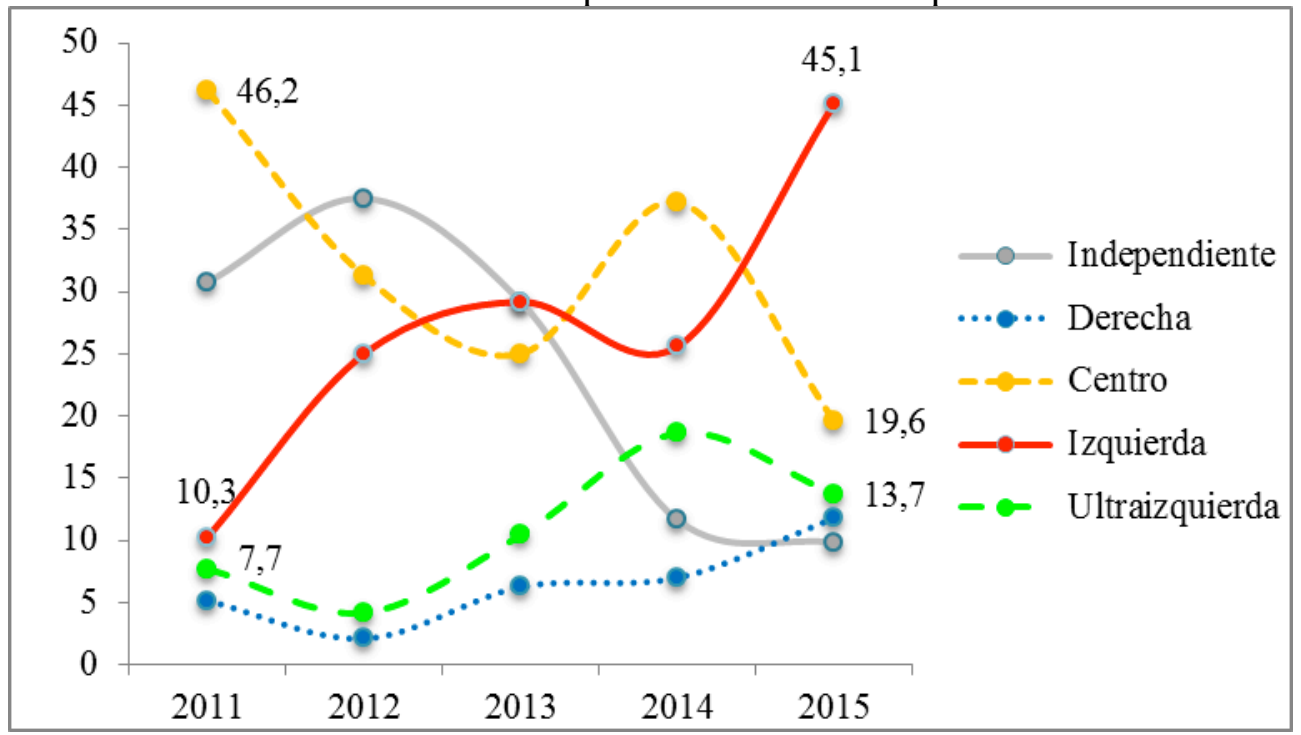

Fuente: Elaboración en base a Caplow (1974) e informes de organizaciones políticas y actas CONFECH (2011-2015)

El siguiente cuadro $\left(\mathrm{N}^{\circ} 4\right)$ permite detallar la composición anual del Pleno distinguiendo cinco posiciones, que pueden llegar a constituir bloques, en la medida que los actores de cada posición o sector logren niveles mínimos de articulación y coordinación estratégica. Se aprecia que durante este período la composición de este espacio se estructura con una tríada en la que cada sector ocupa alternativamente el lugar del actor dominante (A), intermedio (B) o subordinado (C); dependiendo del número de federaciones que controla a nivel nacional respecto del total de federaciones integrantes del espacio. Asimismo, siendo $\mathrm{A}>\mathrm{B}>\mathrm{C}$, las diferencias internas de la tríada determinan las posibilidades de formar coalición y sus efectos políticos. Con este argumento se afirma que, no siempre el sector que obtiene mayor porcentaje de federaciones controla el Pleno, más bien, la posición dominante se construye considerando los resultados iniciales y las posibilidades de sumar fuerzas por parte de orgánicas pertenecientes a más de un sector. 
Cuadro $\mathrm{N}^{\circ}$ 4: Composición anual del Pleno en base a cinco posiciones estratégicas

\begin{tabular}{|c|c|c|c|c|c|}
\hline Año & Independiente & Derecha & Centro & Izquierda & Ultraizquierda \\
\hline 2011 & $30,7(\mathrm{~B})$ & 5,1 & $46,2(\mathrm{~A})$ & $10,3(\mathrm{C})$ & 7,7 \\
\hline 2012 & $37,5(\mathrm{~A})$ & 2,1 & $31,3(\mathrm{~B})$ & $25(\mathrm{C})$ & 4,2 \\
\hline 2013 & $29,2(\mathrm{~B})$ & 6,3 & $25(\mathrm{C})$ & $29,2(\mathrm{~A})$ & 10,4 \\
\hline 2014 & 11,6 & 6,9 & $37,2(\mathrm{~A})$ & $25,6(\mathrm{~B})$ & $18,6(\mathrm{C})$ \\
\hline 2015 & 9,8 & 11,8 & $19,6(\mathrm{~B})$ & $45,1(\mathrm{~A})$ & $13,7(\mathrm{C})$ \\
\hline
\end{tabular}

Fuente: Elaboración en base a Caplow (1974) e informes de organizaciones políticas y actas CONFECH (2011-2015)

De este modo, para el año 2011, la composición del Pleno determina, inicialmente, que los actores ubicados en el Centro tengan una posición dominante (A) (46,2\%), los independientes una posición intermedia (B) (30,7\%) y la izquierda una posición subalterna (C) (10,3\%) (Informes de organizaciones políticas, 2011-2015). Considerando estos datos, las orgánicas ubicadas en el Centro tienen incentivos claros para buscar articulación con Independientes $(\mathrm{AB})$ o con la Izquierda (AC). Se descarta como alternativa probable la coalición $\mathrm{BC}$ por constituir una coalición superflua, esto es, sin efectos políticos relevantes.

En el año 2012, la composición inicial establece que los actores ubicados en la posición de Independientes poseen una posición dominante $(A)(37,5 \%)$, las organizaciones pertenecientes al Centro una posición intermedia (B) $(31,3 \%)$ y la Izquierda una posición subalterna (C) (25\%). Este escenario establece condiciones para la pérdida de la posición dominante del Centro porque los Independientes obtienen el mayor porcentaje de federaciones integradas al Pleno (37,5\% contra 31,3\%) (Informes de organizaciones políticas, 2011-2015). En segundo lugar, la distribución de poder inicial del Pleno resulta equivalente a $\mathrm{A}>\mathrm{B}>\mathrm{C}$, donde $\mathrm{A}<(\mathrm{B}+\mathrm{C})$, por lo cual, la coalición $\mathrm{BC}$ (Coalición Centro e Izquierda) tiene la capacidad de subvertir la configuración inicial del espacio. En tercer lugar, se debe considerar que el sector inicialmente dominante (Independientes), por su propia naturaleza, posee un bajo nivel de cohesión interna que le impide actuar como un actor propiamente tal. Si esto es así, la Izquierda, siendo la tercera mayoría del Pleno tendría una posibilidad privilegiada para constituir coalición, considerando que dispone, por proximidad ideológica, del apoyo de la ultraizquierda y de ciertos actores ubicados en el sector de independientes para constituirse en un actor clave de intermediación.

Para el 2013, por primera vez durante este período, las organizaciones ubicadas en el sector de la Izquierda ocupan una posición dominante inicial (A) (29,2\%), el sector de los Independientes ocupa una posición intermedia (B) $(29,2 \%)$ y el Centro ocupa una posición subalterna (C) (25\%) (Informes de organizaciones políticas, 2011-2015). Se da el caso que en la distribución de poder inicial, la Izquierda (A) y los Independientes (B) alcanzan igual número de federaciones integrantes del Pleno (29,2\% cada uno), generando una correlación inicial del tipo $\mathrm{A}=\mathrm{B}, \mathrm{B}>\mathrm{C}$. Hemos designado a los independientes como posición intermedia (B), debido a que, tal como se ha señalado, su fragmentación interna restringe su agentividad (agency). ${ }^{4}$ Por otra parte, la Izquierda posee una reserva de apoyo

\footnotetext{
${ }^{4}$ Para Adam Przeworski (2010) la agentividad se define como capacidad de acción en el contexto de una democracia representativa, facultad que depende de los grados de efectividad y legitimidad del actor (Przeworski, 2010: 227).
} 
en el sector de la Ultraizquierda de $10,4 \%$ que le da ventaja estratégica sobre el resto de los sectores.

La composición inicial del Pleno para el año 2014 establece que el Centro posee la condición de dominante (A) $(37,2 \%)$, la Izquierda detenta la posición intermedia (B) $(25,6 \%)$ y la Ultraizquierda la posición subalterna (C) $(18,6 \%)$ (Informes de organizaciones políticas, 2011-2015). La distribución inicial de poder del Pleno corresponde a $\mathrm{A}>\mathrm{B}>\mathrm{C}, \mathrm{A}<$ $(\mathrm{B}+\mathrm{C})$, lo que significa que la coalición $\mathrm{BC}$ es de carácter revolucionario, por cuanto subvierte el status de A como dominante en la tríada original. Es necesario agregar que el 2014 las orgánicas correspondientes al Centro se transforman en juventudes políticas oficialistas, adscritas a la Nueva Mayoría, al asumir su segundo gobierno Michelle Bachelet. Este hecho traza una fractura estratégica importante en el espacio CONFECH entre el Centro y la Izquierda, lo que genera incentivos para la formación de coalición entre la Izquierda y la Ultraizquierda, ésta última convertida en tercera fuerza (C) de la tríada predominante del Pleno.

Finalmente, en el año 2015 la composición inicial del Pleno determina que la Izquierda alcanza una holgada posición dominante (A) $(45,1 \%)$, el Centro ocupa la posición Intermedia (B) (19,6\%) y la Ultraizquierda la posición subalterna (C) (13,7\%) (Informes de organizaciones políticas, 2011-2015). Con una distribución de poder $A>B>C, A>(B+C)$, cualquier coalición podría resultar superflua e innecesaria para la Izquierda si no fuera por la posibilidad de fortalecer su convergencia con la Ultraizquierda y generar mayor castigo sobre las organizaciones representativas de la Nueva Mayoría. La necesidad de distanciarse y aislar al Centro, determina claros incentivos para la formación de una coalición AC, con efectos impropios, debido a que rompe la cadena jerárquica interna de la tríada al potenciar la fuerza de la Ultraizquierda sobre el poder de las organizaciones vinculadas al Centro.

Cuadro 5: Coaliciones posibles y probables en el Pleno (Coaliciones probables en negrita) Coaliciones posibles

\begin{tabular}{|c|c|c|c|c|}
\hline Año & Distribución poder & Conservadoras & Revolucionarias & Impropias \\
\hline 2011 & $\mathrm{~A}>\mathrm{B}>\mathrm{C}, \mathrm{A}>(\mathrm{B}+\mathrm{C})$ & $\mathbf{A B}, \mathrm{BC}$ & & $\mathbf{A C}$ \\
\hline 2012 & $\mathrm{~A}>\mathrm{B}>\mathrm{C}, \mathrm{A}<(\mathrm{B}+\mathrm{C})$ & $\mathrm{AB}$ & $\mathbf{B C}$ & $\mathbf{A C}$ \\
\hline 2013 & $\mathrm{~A}=\mathrm{B}, \mathrm{B}>\mathrm{C}$ & $\mathbf{A B}$ & $\mathrm{AC}, \mathrm{BC}$ & \\
\hline 2014 & $\mathrm{~A}>\mathrm{B}>\mathrm{C}, \mathrm{A}<(\mathrm{B}+\mathrm{C})$ & $\mathrm{AB}$ & $\mathbf{B C}$ & $\mathrm{AC}$ \\
\hline 2015 & $\mathrm{~A}>\mathrm{B}>\mathrm{C}, \mathrm{A}>(\mathrm{B}+\mathrm{C})$ & $\mathrm{AB}, \mathrm{BC}$ & & $\mathbf{A C}$ \\
\hline
\end{tabular}

Fuente: Elaboración en base a Caplow (1974) e informes de organizaciones políticas y actas CONFECH (2011-2015)

En base a cada una de las distribuciones iniciales de poder se generan oportunidades de formar coaliciones entre sectores y actores cuyos efectos contribuyen a reestructurar nuevas correlaciones de fuerza. No obstante, las distribuciones de poder en cada año permiten determinar si las alternativas de coalición son conservadoras, revolucionarias o impropias por la capacidad de cada una de mantener o subvertir la composición inicial de este espacio.

Un aspecto relevante que aparece al examinar el periodo, consiste en la importante función del tercer actor, quién en cinco de siete coaliciones probables está presente. Si se observa el aumento en la capacidad de presión en la Izquierda durante 2011 y 2012, así como el mismo aumento en la Ultraizquierda durante 2014 y 2015, se podrá concluir que el 
posicionamiento de las organizaciones adscritas a ambos sectores ocurre en calidad de terceros ganadores (tertius gaudens en Simmel) (2014). El sector de los terceros en la composición inicial del pleno CONFECH durante este lapso, constituye una posición de gran efectividad estratégica ya sea a través de coaliciones impropias que rompen la jerarquía interna de la triada (por ejemplo, durante 2011, 2012 y 2015) y subordina al sector intermedio (B) o a través de coaliciones revolucionarias (por ejemplo, 2012 y 2014) que rompen la jerarquía interna y subordina a sector inicialmente dominante en el Pleno.

\section{CONCLUSIONES}

Como se ha señalado, para el período 2011 a 2015 la variación de la composición del Pleno cambia anualmente dependiendo de los resultados en las elecciones de las federaciones adscritas a CONFECH y con creciente presión sobre la multifederativa, por la centralidad del tema educacional en la agenda pública. Entre las principales tendencias en la composición del espacio se observa: i) disminución de federaciones independientes, ii) disminución de federaciones pertenecientes al centro, iii) aumento significativo en el porcentaje de federaciones de izquierda, iv) aumento moderado de las federaciones pertenecientes a la ultraizquierda, y v) aumento moderado de las federaciones de derecha. Aunque la ultraizquierda y la derecha crecen moderadamente en relación al total de integrantes del Pleno, la mayor proximidad ideológica de la ultra con organizaciones de otros sectores permite mayor capacidad de coalición (especialmente con la izquierda) y por extensión mayor capacidad de chantaje o presión.

Un fenómeno evidente en el estudio de la composición del Pleno durante estos cinco años, radica en la pérdida de influencia de los partidos políticos con representación parlamentaria. La dimensión más inmediata de este fenómeno ha sido la retracción desde el 2011 de la JJCC y el ascenso del bloque de conducción como alianza de tres actores (FEL, IA, UNE), que permite cumplir con la función de intermediación en el espacio y el control necesario sobre las expresiones más radicales del movimiento. Sin embargo, este recambio plantea la interrogante respecto de los modos de producción de conocimiento para la acción y eventuales asimetrías en este aspecto entre diferentes actores de un mismo sector y las consecuencias de ello en la discusión sobre políticas sectoriales. Asimismo, como consecuencia de estas tendencias se constata un creciente nivel de autonomización del espacio respecto de partidos tradicionales, derivado del predominio de fuerzas políticas con propensión a la autolimitación; lo que equivale a menor penetración de estos actores en el espacio estudiantil, pero en sentido opuesto, mayores dificultades para influir desde la CONFECH sobre el gobierno.

Entre los principales efectos de las correlaciones anuales se cuentan: i) la incapacidad del actor dominante inicial para consolidar su condición, ii) el fortalecimiento gradual de actores intermedios y subalternos en la triada que controla el espacio, iii) la propensión a aislar al centro por parte de la izquierda y la ultra-izquierda, iv) la consolidación de la ultra-izquierda como tercer actor posicionado como tertius gaudens.

En cuanto al diseño de estrategias para la formación de alianzas desde las correlaciones anuales del Pleno se puede sostener que las composiciones iniciales del espacio generan los siguientes escenarios probables:

a) Para el año 2011 surgen dos alianzas como escenarios más probables, considerando que $\mathrm{A}=\mathrm{Centro}, \mathrm{B}=$ Independientes y $\mathrm{C}=$ Izquierda: la coalición del Centro con Independientes 
$(\mathrm{A}+\mathrm{B})>\mathrm{C}$ que posee carácter conservador o la coalición del Centro con la Izquierda $(\mathrm{A}+\mathrm{C})>\mathrm{B}$ que posee carácter impropio. En el caso de la coalición conservadora, el Centro mantiene el poder con la colaboración de los Independientes y se anula la presión de la Izquierda por ejercer mayor poder. Por su parte, en la coalición impropia, el Centro mantiene el poder con la colaboración de la Izquierda, excluyendo a los Independientes.

b) Para el año 2012 surgen también dos alianzas como escenarios más probables, considerando que $\mathrm{A}=$ Independientes, $\mathrm{B}=$ Centro y $\mathrm{C}=$ Izquierda: la coalición del Centro con la Izquierda $(\mathrm{B}+\mathrm{C})>\mathrm{A}$ que posee carácter revolucionaria o la coalición de Independientes con la Izquierda $(\mathrm{A}+\mathrm{C})>\mathrm{B}$ que pose carácter de impropia. En la coalición revolucionaria, se generan condiciones para subvertir el poder de los Independientes, a la sazón mayoritarios, mientras tanto, en la coalición impropia se excluye al Centro, subordinándolo a la Izquierda.

c) Para el año 2013 surge una alianza como escenario más probable, considerando que $\mathrm{A}=$ Izquierda, $\mathrm{B}=$ Independientes $\mathrm{y} \mathrm{C}=$ Centro: la coalición de la Izquierda con Independientes $(\mathrm{A}+\mathrm{B})>\mathrm{C}$ posee carácter conservadora. En esta situación, la coalición conservadora genera condiciones para mantener a la Izquierda como actor dominante, excluyendo al Centro.

d) Para el 2014 surge una alianza como escenario más probable, considerando que $\mathrm{A}=$ Centro, $\mathrm{B}=$ =Izquierda y $\mathrm{C}=$ Ultra-izquierda: la coalición de la Izquierda con la Ultraizquierda $(B+C)>A$ posee carácter revolucionaria. En base a este escenario, la coalición revolucionaria genera condiciones para subvertir el poder numérico del Centro, mayoritario en el momento inicial, subordinándolo a la Izquierda, sector que procedería, de este modo, a controlar el espacio.

e) Por último, para el 2015 surge una alianza como escenario más probable, considerando que $\mathrm{A}=$ Izquierda, $\mathrm{B}=$ Centro y $\mathrm{C}=$ Ultra-izquierda: la coalición de la Izquierda con la Ultraizquierda $(\mathrm{A}+\mathrm{C})>\mathrm{B}$ posee carácter impropia. A partir de dicha coalición impropia se producen condiciones para que la izquierda controle el Pleno y, al mismo tiempo, la Ultraizquierda adquiera poder sobre el Centro.

La sucesión de estos escenarios muestra que el actor dominante en la composición inicial del Pleno, generalmente tiene una capacidad limitada de mantener esta condición. Como se aprecia en la composición de los años 2012 y 2014, respectivamente, Independientes y Centro, pierden la condición inicial de dominantes. En cambio, los años 2013 y 2015 la izquierda con el apoyo estratégico de la Ultraizquierda logra mantener el control del espacio. Asimismo, los datos de estos cinco años permiten sostener que la estrategia más eficiente es la desarrollada por los terceros actores de la tríada. El 2011 y 2012, la izquierda logra posicionarse y, de esta manera, dominar el espacio el 2013. Por su parte, durante los años 2014 y 2015 la Ultraizquierda, no solo consolida su posición de tercero, sino que, al sostener la condición de dominante de la Izquierda, contribuye de manera decisiva a la exclusión y aislamiento de las orgánicas pertenecientes al Centro del espacio de la multifederativa.

SANTIAGO (CHILE), FEBRERO 2016 
RECIBIDO: FEBRERO 2016

ACEPTADO: ABRIL 2016

\section{REFERENCIAS BIBLIOGRÁFICAS}

\section{Libros y artículos}

AGACINO, R. (2013). "Movilizaciones estudiantiles en Chile. Anticipando el futuro". Educação en Revista Marília, Vol. 14, n. 1.

AVENDAÑO, O. (2014). "Fracturas y representación política en el movimiento estudiantil: Chile 2011". Última Década 22(41). Valparaíso: CIDPA.

BOURDIEU, P. (1985). ¿Qué significa hablar? Economía de los intercambios lingüisticos. Madrid, AKAL.

---- --- (1999). Intelectuales, política y poder. Buenos Aires, EUDEBA.

CAPLOW, T. (1974) Dos contra uno: teoría de las coaliciones en las tríadas. Madrid, Alianza Editorial.

CHAISTY, P.; CHEESEMAN, N., \& POWER, T. (2012). "Rethinking the 'Presidentialism Debate': Conceptualizing Coalitional Politics in Cross-Regional Perspective". Democratization. Londres: Routledge. Disponible en:

http://www.tandfonline.com/doi/pdf/10.1080/13510347.2012.710604

GARCÉS, M. (2012). El despertar de la sociedad. Santiago de Chile: LOM ediciones.

GARRETÓN, M. (1984). El movimiento estudiantil: conceptos e historia. Biblioteca del Movimiento Estudiantil. Tomo IV. Santiago de Chile: Ediciones SUR.

---- ---- (2011). "Movimiento social, nuevas formas de hacer política y enclaves autoritarios. Los debates del Consejo Asesor para la Educación en el gobierno de Michelle Bachelet en Chile". Polis $N^{\circ} 10$.

HELMKE, G. Y LEVITSKY, S. (2003) "Informal institutions and comparative politics: a research agenda". Perspectives on Politics. Volume 2 / Issue 04 / December 2004, 725-740. American Political Science Association DOI: http://dx.doi.org/10.1017/S1537592704040472 (About DOI), Published online: 01 December 2004

MAYOL, A Y AZÓCAR, C. (2011). "Politización del malestar, movilización social y transformación ideológica: el caso Chile 2011". Polis $\mathrm{N}^{\circ} 10$.

MELlA, M. (2012). Elementos de Ciencia Política. Conceptos, Actores y Procesos. Vol. 1. Santiago: RIL.

---- ---- (2012). "Efectos sociales del terremoto en chile y gestión política de la reconstrucción durante el gobierno de Sebastián Piñera (2010-2011)”. Revista Enfoques. Ciencia Política y Administración Pública. Santiago.

MELLA, MARCELO; RIVERA, RICARDO; RÍOS, HÉCTOR (2016). "Condiciones orgánicas y correlaciones de fuerza del movimiento estudiantil chileno. Una aproximación desde la Confech (2011 - 2015”). Revista Izquierdas. Disponible en: http://www.redalyc.org/articulo.oa? id=360144967006

MOULIAN, T. (2002). Chile Actual. Anatomía de un mito. Santiago: LOM.

MUÑOZ, V. (2009). "Generaciones en tránsito: Juventud universitaria e izquierdas políticas en Chile y México (Universidad de Chile- UNAM 1984-2006)”. Tesis doctoral. México: Universidad Nacional Autónoma de México. 
MUNCK, G. (1995). “Algunos problemas conceptuales en el estudio de los movimientos sociales". Revista Mexicana de Sociología. Vol. 57, No. 3.

---- ---- (1997). "Formação de Atores, Coordenação Social e Estratégia Política: Problemas Conceituais hacen Estudo dos Movimentos Sociais". Revista Dados, 40 (1).

ROSSI, J. (2012). "La movilización estudiantil chilena en 2011: una cronología". OSAL Observatorio Social de América Latina, Año 13, N³1. Disponible en: http://biblioteca.clacso.edu.ar/clacso/osal/20120417105250/OSAL31.pdf

PRZEWORSKI, A. (2010), Qué esperar de la democracia. Limites y posibilidades del autogobierno. Buenos Aires: Siglo XXI.

SALAZAR, G. (2012). Movimientos sociales en Chile. Santiago de Chile: Uqbar editores.

SARTORI, G. (1980). Partido y sistemas de partido. Marco para un análisis. Madrid: Alianza.

SIMMEL, G. (2014). Sociología: estudios sobre las formas de socialización. México D.F., FCE.

TAAGEPERA, R. (2007). "Electoral Systems". En Boix, C. y Stokes, S. The Oxford Handbook of Comparative Politics. Nueva York: Oxford University Press Inc.

TEJERINA, B. (2005). "Movimientos sociales, espacio público y ciudadanía: Los caminos de la utopía". Revista Crítica de Ciencias Sociales, $\mathrm{N}^{\circ} 72$.

THIELEMANN, L. (2011). "Para una periodificación del movimiento estudiantil de la transición (1987-2011)”. En: Sistematización de Talleres para la Acción Estudiantil. CEFECH-Heinrich-Böll-Stiftung. Santiago.

\section{Documentos públicos}

CONFECH (2010) Reglamento de Sala. Aprobado en la Sesión Ordinaria de 15 de agosto de 2010 realizada en la Universidad de Santiago de Chile. Recuperado de: http://movimientoestudiantil.cl/wp-content/uploads/2015/12/2010-Reglamento-deSala-CONFECH-1.pdf

CONFECH (2015) Propuesta de reglamento de Sala. Aprobada en la Sesión Ordinaria de 10 de enero de 2015, realizada en la Universidad Arturo Prat de Iquique.

CONFECH (2011-2015) Sintesis y Actas Sesiones, desde el 16 de abril de 2011 al 12 de septiembre de 2015.

\section{Documentos privados}

Informes de organizaciones políticas (2011-2015) sectores centro, izquierda y ultraizquierda.

\section{Entrevistas}

Mella M. Ríos H. y Rivera R. (2015a) Entrevista a militante organización política perteneciente al sector ultra-izquierda. Santiago, Septiembre 2015.

---- ---- (2015b) Entrevista a presidente federación estudiantes perteneciente al sector ultraizquierda. Santiago, Agosto 2015.

---- ---- (2015c) Entrevista a militante organización política perteneciente al sector izquierda. Santiago, Noviembre 2015.

---- ---- (2014) Entrevista a militante organización política perteneciente al sector centro. Santiago, Noviembre 2014. 